\title{
Comportamento Sexual de Surdos Participantes da Comunidade Surda de Maceió
}

\section{Sexual Behavior of Deaf Participants of the Deaf Community of Maceió}

El Comportamiento Sexual de los Participantes Sordos de la Comunidad Sorda de Maceió

Kety Lucy Ferreira da Silva ${ }^{1}$

Sandra Lopes Cavalcanti ${ }^{2}$

\section{Resumo}

Objetivo: Investigar o comportamento sexual dos surdos ativos sexualmente, participantes do grupo instituído como Comunidade Surda de Maceió. Método: pesquisa quantitativa, descritiva, de abordagem transversal, em que se pretende pesquisar o comportamento dos surdos sexualmente ativos com idade acima de 14 anos, sendo participantes do grupo instituído como Comunidade Surda de Maceió, no período de 12 meses. Resultados Esperados: obtenção de dados que confirmem a existência do comportamento sexual de risco dos surdos e suas causas, evidenciando a importância de políticas públicas voltadas para este grupo que visem contribuir com a redução desses riscos.

\section{Descritores: Comportamento Sexual; Sexualidade; Surdez; Doenças Sexualmente Transmissíveis.}

\begin{abstract}
Objective: To investigate the sexual behavior of deaf sexually active, participants in the group called the Deaf Community of Maceió. Method: descriptive quantitative research, transversal approach with the intention of researching the behavior of sexually active deaf over the age of 14 years, those participants of the group called
\end{abstract}


the Deaf Community of Maceio, in the period of 12 months. Expected Results: obtaining data confirming the existence of the sexual risk behavior of the deaf and their causes, highlighting the importance of public policies aimed at this group to contribute with reducing these risks.

Descriptors: $\quad$ Sexual Behavior; Sexuality; Deafness; Disabled Persons; Sexually Transmitted Infections.

\section{Resumen}

Objetivo: Investigar el comportamiento sexual de las personas sordas sexualmente activos participantes en el grupo constituido como comunidad sorda de Maceió. Método: investigación cuantitativa descriptiva, enfoque transversal, en el que desea investigar en el comportamiento de las personas sordas sexualmente activas mayores de 14 años, los miembros del grupo configurado como Comunidad Sorda de Maceió, en el período de 12 meses. Resultados esperados: la obtención de datos que confirmen la existencia de la conducta sexual de riesgo de las personas sordas $y$ sus causas, destacando la importancia de las políticas públicas para este grupo para contribuir a reducir estos riesgos.

\section{Descriptores: Conducta Sexual;} Sexualidad; Sordera; Personas con Discapacidad; Enfermedades de Transmisión Sexual.

\section{Introdução}

Em geral, existe uma preocupação a respeito dos altos índices de gravidez na adolescência registrados a cada ano no Brasil, como também em relação aos casos de Infecções Sexualmente Transmissíveis (ISTs) e essa preocupação se estende ainda mais quanto ao comportamento sexual das pessoas com deficiência, sobretudo dos surdos do grupo instituído como Comunidade Surda, isso porque pouco se sabe acerca do comportamento sexual deles. Há uma escassez de pesquisas acerca do tema a nível mundial, pois as grandes discussões e pesquisas científicas estão relacionadas a questões de língua e cultura $^{(1)}$, entretanto, os poucos estudos nos mostram que os surdos possuem um

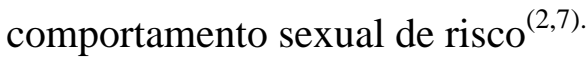

Em 2013 o Brasil registrou 559.991 nascidos vivos de mães com idade entre 10 a 19 anos, sendo 14.146 em Alagoas e 3.672 registros em 
Maceió $^{(8)}$. Dados preliminares do ano de 2014 estima que o Brasil registrou nessa mesma faixa etária, 561.899 nascidos vivos, sendo 13.608 registrados em Alagoas e na capital, 3.479; no município de Maceió, somente 184 nascidos vivos de mães com idade entre 10 a 14 anos foram registrados em $2014^{(8)}$.

Além dos altos índices de gravidez, o Brasil também possui um número significativo de registros de Infecções Sexualmente Transmissíveis; o país registrou, no período entre janeiro a 30 de junho de 2015, 474 casos de gestantes infectadas por HIV, de acordo com ano de parto, também na faixa etária entre 10 a 19 anos. ${ }^{(9)}$. Já em Maceió, apenas 29 casos foram registrados, considerando todas as faixas etárias $^{(10)}$.

Embora não haja dados que indiquem com precisão casos de nascidos vivos de mães com deficiência auditiva que foram infectadas pelo Vírus da Imunodeficiência Humana, é nesse contexto que a Comunidade Surda está inserida, considerando que o censo demográfico de 2010 mostrou que no Brasil, 9,7 milhões de pessoas possuem deficiência auditiva, sendo uma parte delas, consideradas surdas; 186 mil destas estão localizadas no estado de
Alagoas, e em Maceió somam $55.812^{(11)}$.

Verificamos na literatura que o comportamento sexual de risco dos jovens, sem distinção, está atrelado a diversas variantes, entre elas, relatos de múltiplos parceiros ${ }^{(12)}$ e a história de abuso sexual ${ }^{(13-14)}$. As literaturas nos mostram ainda que os métodos preventivos são cada vez menos usados pelos jovens à medida que os relacionamentos vão se consolidando ${ }^{(15)}$, assim os altos índices de gravidez e os casos de contaminação por HIV ou outras Infecções Sexualmente Transmissíveis podem ser justificados por essa mudança de comportamento ocorrida ao longo dos anos, por outro lado, indícios apontam que as pessoas com deficiência iniciam a vida sexual por volta dos 14 anos, ou seja, mais cedo em comparação à população em geral e que em suas histórias, além de terem relatos de violência sexual e envolvimento com múltiplos parceiros, há sexo desprotegido $^{(4,6)}$; isso desencadeia um comportamento sexual bastante preocupante.

No caso da pessoa surda esse comportamento pode ser bem maior devido à limitação existente na comunicação; tendo a língua de sinais como característica primordial, os 
surdos apresentam uma barreira linguística que obstrui a comunicação entre eles e os demais que não compartilham da mesma linguagem dentro da sociedade. Essa barreira pode impedir que as informações acerca da prevenção e dos métodos contraceptivos alcancem-nos e podem levá-los a se comportarem sexualmente de forma inadequada $^{(7,16)}$.

Diante das poucas discussões a respeito do comportamento da pessoa surda, encontramos uma pesquisa realizada em 1995, com alunos surdos de uma universidade em Washington, tal pesquisa constatou que da maioria dos estudantes surdos entrevistados, $11 \%$ se tornaram sexualmente ativos antes da idade de 18, e a primeira relação deu-se aos 14 anos ou menos; a pesquisa também revelou um alto índice de abuso sexual o que pode justificar a iniciação sexual nessa faixa etária ${ }^{(2)}$.

No sul do Brasil, o comportamento sexual das pessoas com deficiência auditiva, com idade acima de 15 anos foi investigado em 2009, ficando constatado que $40 \%$ dos surdos usavam menos preservativo em relação aos ouvintes e $60,6 \%$ destes surdos afirmaram ser impossível contrair $\operatorname{AIDS}^{(3)}$.

Outra pesquisa que englobou aproximadamente 282 surdos, usuários da língua de sinais americana (ASL), com idade entre 19 a 64 anos, realizada na comunidade surda de Rochester mostrou que os surdos possuem um perfil de risco maior em relação à população da cidade, pois apresentou elevada propensão em se envolver com atividades de múltiplos parceiros, além disso, revelou uma prevalência de surdos que mantiveram relacionamentos homoafetivos mesmo estando $\operatorname{casados}^{(5)}$.

Os poucos estudos publicados abordando a surdez e a sexualidade entre pessoas com deficiência auditiva, constataram que o conhecimento da população surda estava muito inadequado $^{(3,7,12)}$, contudo, os mesmos estudos afirmaram que todas as constatações são insuficientes, sugerindo novas pesquisas.

É bem verdade que não sabemos as causas desses comportamentos inadequados, porém, podem estar relacionadas à possibilidade desses sujeitos apresentarem limitações de linguagem e habilidades cognitivas quando isolados da sociedade, assim, irão acessar menos ou usar inadequadamente as medidas preventivas $^{(3)}$; a incapacidade de ouvir pode levar a uma condição de isolamento de informações devido às barrreiras linguísticas existentes na 
sociedade, limitando o conhecimento sobre diversas questões comuns aos de outra população, todavia, tais alegações não podem ser sustentadas porque há pouca literatura a respeito $^{(2)}$, dessa forma, pretendemos investigar $\mathrm{o}$ comportamento sexual dos surdos da cidade de Maceió para verificarmos a existência desse comportamento de risco e suas causas, além de somar dados às constatações existentes.

Nosso objetivo geral será investigar o comportamento sexual dos surdos ativos sexualmente, usuários da Língua Brasileira de Sinais (Libras). E os específicos serão: identificar as causas desse comportamento; identificar os possíveis riscos pertinentes à prática sexual dos surdos e verificar o conhecimento dos surdos sobre a prevenção e os métodos contraceptivos.

\section{Método}

Trata-se de uma pesquisa quantitativa descritiva, de abordagem transversal, em que se pretende pesquisar o comportamento dos surdos sexualmente ativos, com idade acima de 14 anos, participantes do grupo instituído como Comunidade Surda de Maceió, no período de 12 meses.

Serão inclusos todos os surdos com idade a partir de 14 anos, sendo sexualmente ativos e participantes do grupo instituído como Comunidade Surda de Maceió, portanto, usuários da Língua Brasileira de Sinais. Serão excluídos da pesquisa os sujeitos menores de idade, cujos pais não assinarem o Termo de Consentimento Livre e Esclarecido. Serão excluídos ainda, os surdos não ativos da Comunidade; os que possuam algum tipo de transtorno mental e os que não se comunicam através da Libras.

Como instrumento de coleta de dados, será elaborado um questionário contendo questões relacionadas à prática sexual, fonte de informações, conhecimento sobre prevenções e métodos contraceptivos, características familiares (questões sobre o pai, irmãos, companheiro da mãe, qualidade de vida da família, vínculo empregatício, hábitos familiares, violência doméstica e sexual), apenas com opções de marcar X. As perguntas serão apresentadas na modalidade visual (Libras) e modalidade escrita (Português), e obrigatoriamente deverá ser respondida no questionário impresso, em Português.

\section{Resultados}

Verificar se há nesse grupo um comportamento sexual de risco, e em 
caso positivo, constatar as causas desse comportamento inadequado,

evidenciando a importância de políticas públicas voltadas para este grupo que visem contribuir com a redução desses riscos.

A hipótese é que os comportamentos inadequados apresentados pelos surdos participantes da Comunidade Surda estejam relacionados às barreiras linguísticas e/ou, tenham uma relação direta com as histórias de abusos sexuais.

\section{Conclusão}

No momento fica impossível apresentar uma conclusão uma vez que se trata da apresentação do projeto.

\section{Colaborações}

A realização deste trabalho foi possível devido à colaboração da professora Dra. Divanise Suruagy Correia que orientou e contribuiu na revisão do conteúdo.

\section{Agradecimentos}

Agradecemos também ao graduando do Curso de Enfermagem da ENSEFAR-UFAL, Davi Porfírio da
Silva, que contribuiu na coleta dos dados parciais.

\section{Referências}

1. Lebedeff TB. Surdez, sexualidade e diferença: a construção de sentidos na Babel globalizada. In: Júnior FA, Almeida T, organizadores. Sexualidade, cinema e deficiência. São Paulo: Livraria Médica Paulista Editora; 2008; 203-22.

2. Joseph JM, Sawyer R, Desmond S. Sexual Knowledge, behavior and sources of information among deaf and hard of hearing college students. Am Ann Deaf (Maryland) [periódico na internet]. 1995 [cited 2016 Jan. 31]; 140(4): 338-45. Available from: https:// muse.jhu.edu/article/385015.

3. Freire DB, Gigante LP, Béria JU, Palazzo LS, Figueiredo ACL, Raymann BCW. Acesso de pessoas deficientes auditivas a serviços de saúde em cidade do Sul do Brasil. Cad. Saúde Pública (Rio de Janeiro) [periódico na internet]. 2009 [citado 2016 fev. 15]; 25(4):88997. Disponível em: http://www.scielosp. org/pdf/csp/v25n4/20.pdf.

4. Gomez MGA. Sexual behavior among Filipino high school students who are Deaf. Sex. disabil. (New York) [periódico na internet]. 2011 [cited 2016 Feb. 15]; 29 (4): 301-12. Available from: http://link.springer.com/article/10. 1007\%2Fs11195-011-9215-7.

5. Heiman E, Haynes S, McKee M. Sexual health behaviors of Deaf American Sign Language (ASL) users. Disabil. Health J (USA) [periódido na internet]. 2015 [cited 2016 Jan. 20]; 8(4): 579-85. Available from: http://www.disabilityandhealthjnl.com/a rticle/S1936-6574(15)00094-1/abstract. 
6. Umoren AM, Adejumo AO. Role of Sexual Risk Behaviors and Sexual Attitude in Perceived HIV Vulnerability Among Youths with Disabilities in Two Nigerian Cities. Sex. disabil. (New York) [periódico na internet]. 2014 [cited 2016 Jan. 23]; 32(3): 323-34. Available from: http://link.springer.com /article/10.1007\%2Fs11195-014-93664.

7. Tobin-West C, Akani Y. Human Immuno-Deficiency Virus Information: The Challenges of Hearing-Impaired Adolescents in Port Harcourt, Nigeria. Sex. disabil. (New York) [periódico na internet]. 2014 [cited 2016 jan. 18]; 32 (3): 299-309. Available from: http://link.springer.com/article/10.1007 \%2Fs11195-014-9362-8.

8. Ministério da Saúde (BR) [na internet]. Secretaria Executiva. Datasus. [citado 2016 jun. 11]. Informações de Saúde. Estatísticas vitais. Disponível em: http://www.datasus.gov.br.

9. Ministério da Saúde (BR) [internet]. Secretaria de Vigilância em Saúde. [citado 2016 jun.11]. Departamento de DST, AIDS e Hepatites Virais. HIV em gestantes, Aids e Mortalidade por Aids. Disponível em: http://www.aids.gov.br/.

10. Ministério da Saúde (BR) [internet]. Secretaria de Vigilância em Saúde. [citado 2016 jun. 11]. Departamento de DST, AIDS e Hepatites Virais. Indicadores e dados básicos do HIV/AIDS dos municípios brasileiros. Disponível em: http://svs.aids.gov.br/ai ds/.

11. Instituto Brasileiro de Geografia e Estatística (BR) [internet]. Características gerais da população, religião e pessoas com deficiência, censo 2010. [citado 2016 jun. 11]. Disponível em: http://www.ibge.gov.br/
12. Job J. Factors involved in the ineffective dissemination of sexuality information to individuals who are deaf or hard of hearing. American Am Ann Deaf (Maryland) [periódico na internet]. 2004 [cited 2016 mar. 30]; 149(3): 26473. Available from: https://muse.jhu.edu /article/173623.

13. Poulin C, Graham L. The association between substance use, unplanned sexual intercourse and other sexual behaviors among adolescent students. Addiction (Abingdon) [periódi co na internet]. 2001 [cited 2016 mar. 12]; 96(4):607-21. Available from: http://onlinelibrary.wiley.com/doi/10.10 46/j.13600443.2001.9646079.x/abstract.

14. Cruzeiro ALS, Souza LDM, Silva RA, Pinheiro RT, Rocha CLA, Horta BL. Comportamento sexual de risco: fatores associados ao número de parceiros sexuais e ao uso de preservativo em adolescentes. Ciênc. Saúde Colet. (Rio de Janeiro) [periódico na internet]. 2010 [citado 2016 fev. 21]; 15(supl 1): 1149-58. Disponível em: http://www.scielo.br/scielo.php?script=s ci_arttext\&pid=S1413-812320100007 $00023 \& \operatorname{lng}=$ pt\&nrm=iso\&tlng $=$ pt.

15. Paiva V, Calazans G, Venturi G, Dias R. Idade e uso de preservativo na iniciação sexual de adolescentes brasileiros. Rev Saúde Pública (São Paulo) [periódico na internet]. 2008 [citado 2016 fev. 21]; 42(Supl 1 ): 4553. Disponível em: http://www.scielo.br /scielo.php?script=sci_arttext\&pid=S00 $3489102008000800007 \& \operatorname{lng}=$ pt\&nrm $=\mathrm{i}$ sso.

16. Mprah WK. Knowledge and use of contraceptive methods amongst deaf people in Ghana. Afri J disabil. (Africa) [periódico na internet]. 2013 [cited 2016 feb. 22]; 2(1): 1-9. Available from: http://www.ajod.org/index.php/ajod/arti cle/view/43. 